\title{
Stromal interaction molecule 1 plays an important role in gastric cancer progression
}

\author{
JIN-MEI XU* ${ }^{*}$ YAN ZHOU* ${ }^{*}$ LONG GAO, SHU-XIAN ZHOU, WEI-HUA LIU and XIAO-AN LI \\ The Gastroenterology Tumor and Microenvironment Laboratory, Department of Gastroenterology, \\ The First Affiliated Hospital of Chengdu Medical College, Chengdu Medical College, \\ Xindu, Chengdu, Sichuan 610041, P.R. China
}

Received November 23, 2015; Accepted December 27, 2015

DOI: $10.3892 /$ or.2016.4704

\begin{abstract}
Studies have shown that stromal interaction molecule 1 (STIM1) is expressed in a variety of cancers and is related to tumor growth. The present study aimed to investigate the expression and roles of STIM1 in gastric carcinoma. Immunohistochemistry and western blotting revealed that STIM1 was expressed at higher levels in gastric cancer tissues $(82 \%)$ than these levels in normal gastric tissues (42\%). In addition, STIM1 was also expressed in tumor vascular endothelial cells. The effects of STIM1 on proliferation, apoptosis, adhesion, invasion and migration of gastric cancer cells were detected by MTT assay, flow cytometry, cell adhesion assay and Transwell assay, respectively. The results shown that STIM1 knockdown did not alter proliferation or apoptosis, but promoted cell adhesion and inhibited migration and invasion in the gastric cancer cells. In addition, STIM1 knockdown did not alter the expression or phosphorylation of mitogen-activated protein kinase (MEK) or extracellular signal-regulated kinase (ERK), implying that STIM1 affected gastric cancer cell migration through a pathway independent of the MEK/ ERK pathway.
\end{abstract}

\section{Introduction}

Gastric cancer is one of the most common malignant tumors, with more than 1 million new cases diagnosed worldwide in 2012. Nearly two-fifths of these new cases were diagnosed in

Correspondence to: Professor Xiao-An $\mathrm{Li}$ or Professor Wei-Hua Liu, Gastroenterology Tumor and Microenvironment Laboratory, Department of Gastroenterology, The First Affiliated Hospital of Chengdu Medical College, Chengdu Medical College, 4 Baoguang Road, Xindu, Chengdu, Sichuan 610041, P.R. China

E-mail: zqzy1983@163.com

E-mail: 872485363@qq.com

*Contributed equally

Key words: stromal interaction molecule 1, gastric tumor, storeoperated calcium entry, store-operated calcium channel, metastasis patients in mainland China. Moreover, gastric cancer accounts for $\sim 9 \%$ of all cancer-related deaths $(\sim 723,000 /$ year $)$ (1). Tumor metastasis is the direct cause of death for the majority of patients with gastric cancer. Therefore, therapies that inhibit the invasion and metastasis of tumors are a primary focus of cancer researchers.

$\mathrm{Ca}^{2+}$ is a critical regulator of cell migration in various cell types, including tumor cells (2), and controls a broad range of cellular functions, such as gland secretion, neural excitation, muscle contraction and apoptosis $(2,3)$. Store-operated calcium influx is the dominant mechanism through which $\mathrm{Ca}^{2+}$ enters non-excitable cells, including tumor cells $(4,5)$. Recent studies have shown that stromal interaction molecule 1 (STIM1) and Orail are involved in store-operated $\mathrm{Ca}^{2+}$ entry. While Orai1 is an essential channel protein, STIM1 is a 77-kDa, endoplasmic, single-pass membrane protein that functions to maintain intracellular calcium homeostasis and regulate intracellular calcium ion concentrations (6). Indeed, the SOCE channel, which consists of STIM1 and Orai1, is a major route of $\mathrm{Ca}^{2+}$ entry in non-excitable cells $(6,7)$. STIM1 has mostly been implicated in functions in the immune system (8-10) and cardiovascular system $(11,12)$. Recent studies have also shown that STIM1 is expressed in a variety of cancers, including breast (13), cervical (14), liver (15) and colon cancer (16). Additionally, STIM1 knockdown has been shown to accelerate motility in melanoma cells $(17)$. In contrast, Yang et al $(13,15)$ demonstrated that silencing of STIM1 or Orail inhibited the migration and metastasis of breast cancer and hepatocellular carcinoma. STIM1 has also been shown to mediate cell proliferation and apoptosis in a variety of cell lines. However, STIM1 does not affect apoptosis in breast cancer cells, demonstrating that STIM1 may have cell type-specific effects on cancer phenotypes. Additionally, the role of STIM1 in gastric cancer has not been established.

Therefore, in the present study, we examined the expression of STIM in gastric cancer tissues and evaluated the role of STIM1 in promoting cancer-associated malignant behaviors in gastric cancer cells.

\section{Materials and methods}

Cell culture and RNA interference. Human gastric cancer cells (MKN-45, SGC7-901, AGS and NCI-N87 cells) were donated 
by the National Key Laboratory of Biotherapy. Cells were maintained in Roswell Park Memorial Institute (RPMI)-1640 medium supplemented with $10 \%$ fetal bovine serum (FBS) (both from HyClone) in a humidified atmosphere with $5 \% \mathrm{CO}_{2}$ at $37^{\circ} \mathrm{C}$ for $24 \mathrm{~h}$. Small interfering RNA (siRNA) containing the target sequence for STIM1 (5'-GGUUUGCCUA UAUCCAGAACCGUUA-3' and 5'-UAACGGUUCUGGAUA UAGGCAAACC-3') or control siRNA (Invitrogen, Carlsbad, CA, USA) was transfected into human gastric cancer cells. At 24 or $48 \mathrm{~h}$ following transfection, the cells were treated and prepared for cell migration, adhesion and invasion assays.

Collection of clinical specimens and immunohistochemical analysis. Ninety gastric tumor tissues and 30 normal gastric tissues were collected from the First Affiliated Hospital of Chengdu Medical College (Chengdu, China). The protocol of the present study was approved by the Institutional Ethics Committee of Chengdu Medical College. Informed consent for the present study was received from all patients prior to the commencement of the experiments. The tissues were preserved in $4 \%$ polyoxymethylene solution, embedded in paraffin and sectioned. Endogenous peroxidase was blocked by incubation for $15 \mathrm{~min}$ in $0.3 \% \mathrm{H}_{2} \mathrm{O}_{2}$. Antigen retrieval was carried out in $0.01 \mathrm{M}$ sodium citrate-hydrochloric acid buffer solution. Monoclonal mouse anti-STIM1 antibodies $(4 \mu \mathrm{g} / \mathrm{ml}$; Abcam, Cambridge, UK) were used for immunohistochemical analysis of STIM1 expression for all samples. Monoclonal mouse anti-CD31 antibodies (1:1,000; Epitomics, Burlingame, CA, USA) were used for analysis of the expression of CD31 in vascular endothelial cells of the tissue samples. Tissues were incubated with the primary antibody at $4^{\circ} \mathrm{C}$ overnight. After washing with phosphate-buffered saline (PBS), the cells were incubated with the appropriate secondary antibody (goat anti-mouse $\mathrm{IgG}$ ) for $30 \mathrm{~min}$ at $37^{\circ} \mathrm{C}$. Peroxidase activity was revealed by 3,3-diaminobenzidine and cells were counterstained with hematoxylin. Negative controls were processed with isotype control antibody. Saturation and intensity of the immunostained cells were evaluated over eight visual fields under a light microscope (Olympus Optical, Tokyo, Japan). For statistical analysis, total staining of the protein of interest was scored as the product of the staining intensity (on a scale of 0-3: negative, 0 ; weak, 1; moderate, 2; and strong, 3) x the percentage of cells stained (positively recorded on an ordered categorical scale: 0 , zero; $1,1-25 \% ; 2,26-50 \%$; and 3, 51-100\%), resulting in a scale of $0-9$. The evaluation was performed by two independent investigators.

Western blot analysis. Total protein was extracted from the lysed cells or tissues ground in liquid nitrogen. Protein samples were then subjected to sodium dodecyl sulfate-polyacrylamide gel electrophoresis and transferred to polyvinylidene difluoride membranes. The membranes were blocked in 5\% non-fat milk for $1 \mathrm{~h}$ and then incubated overnight with primary mouse anti-STIM1 antibodies $(2 \mu \mathrm{g} / \mathrm{ml})$ at $4^{\circ} \mathrm{C}$ overnight. The dilution of the antibodies against extracellular signal-regulated kinase (ERK), mitogen-activated protein kinase (MEK), phospho-ERK1/2, p-MEK (Abcam) and $\beta$-actin (SigmaAldrich, St. Louis, MO, USA) was 1:1,000. The membranes were then incubated with a 1:20,000 dilution of peroxidaselinked anti-rabbit or anti-mouse IgG secondary antibodies for $2 \mathrm{~h}$ at $37^{\circ} \mathrm{C}$, and proteins were visualized using BeyoECL Plus (both from Beyotime, Guangdong, China). The intensity of the immunoreactive bands was analyzed using ImageJ and normalized to the expression of $\beta$-actin. Western blotting was used to explore the levels of ERK, MEK, p-ERK, p-MEK expressed in the MKN-45 human gastric cancer cells.

Reverse transcription-polymerase chain reaction (RT-PCR). Total RNA was extracted from human stomach tissues and cell lines with TRIzol reagent (Invitrogen). A NanoDrop 2000 was used to measure the concentration and quality of RNA. The total RNA was then reverse transcribed into cDNA, followed by PCR using a PrimeScript RT-PCR kit (Takara Bio, Shiga, Japan) according to the manufacturer's instructions. Quantitative real-time PCR was performed using SYBR Premix Ex Taq (Perfect Real-Time) (Takara code: DRR041) according to the manufacturer's instructions. The following primer sets were used for evaluation of STIM1 expression: STIM1 forward, 5'-TGTGTCTCCCTTGTCCATGC-3' and reverse, 5'-CATCTGAGGTTTGGGGG-3'; GAPDH forward, 5'-AGGGCTGCTTTTAACTCTGGT-3' and reverse, 5'-CCC CACTTGATTTTGGAGGGA-3'. The relative differences in expression of STIM1 mRNA were calculated using the comparative cycle threshold method.

Flow cytometry. Gastric cancer cells were seeded into 24-well plates and incubated at $37^{\circ} \mathrm{C}$ until reaching $60-70 \%$ confluency. The cells were transfected with STIM1-siRNA for 24 or $48 \mathrm{~h}$ and then harvested using trypsin. The cells were collected in 1.5-ml centrifuge tubes, washed with PBS and resuspended in $1 \mathrm{X}$ binding buffer. Next, $2 \mu \mathrm{l}$ of Annexin $\mathrm{V}$ was added, followed by addition of $2 \mu \mathrm{l}$ of propidium iodide (PI). Cells were incubated at room temperature for $30 \mathrm{~min}$ in the dark. Cell apoptosis was then determined by flow cytometry (BD Biosciences, Franklin Lakes, NJ, USA). Approximately 10,000 events (cells) were evaluated for each sample.

Cell proliferation. Cell proliferation was determined by MTT assay. Cells were transfected with STIM1-siRNA for 24 or $48 \mathrm{~h}$. Ten microliters of $10 \mathrm{mg} / \mathrm{ml} \mathrm{3-[4,5-dimethylthiazol-2-yl]-}$ 2,5-diphenyltetrazolium bromide (MTT) was added to each well, and the plates were incubated at $37^{\circ} \mathrm{C}$ under $5 \% \mathrm{CO}_{2}$ for $4 \mathrm{~h}$. The supernatant was removed, and the formed formazan crystals were dissolved in $100 \mu$ l dimethyl sulphoxide. The absorbance at $450 \mathrm{~nm}$ was then measured with a BD microplate reader. Growth inhibition was determined from the differences in optical densities between the experimental and control wells as a percentage of the control.

Wound healing assay. Cells ( $2 \times 10^{5}$ cells $\left./ \mathrm{ml}\right)$ were seeded in each well of a $24-w e l l$ plate and incubated at $37^{\circ} \mathrm{C}$ until reaching $60-70 \%$ confluency. The cell monolayer was scratched with a $10-\mu 1$ pipette tip, and the cells were then washed with PBS and transfected with STIM1-siRNA. After 48 h, randomly chosen fields were photographed under a light microscope with a $4 \mathrm{x}$ objective.

Migration and invasion assays. Migration assays were carried out using Transwell plates (24-well plates, 8 -mm pore size; Corning, Shanghai, USA). Cells $\left(10^{5} / 0.2 \mathrm{ml}\right)$ were trypsinized, 
A

需
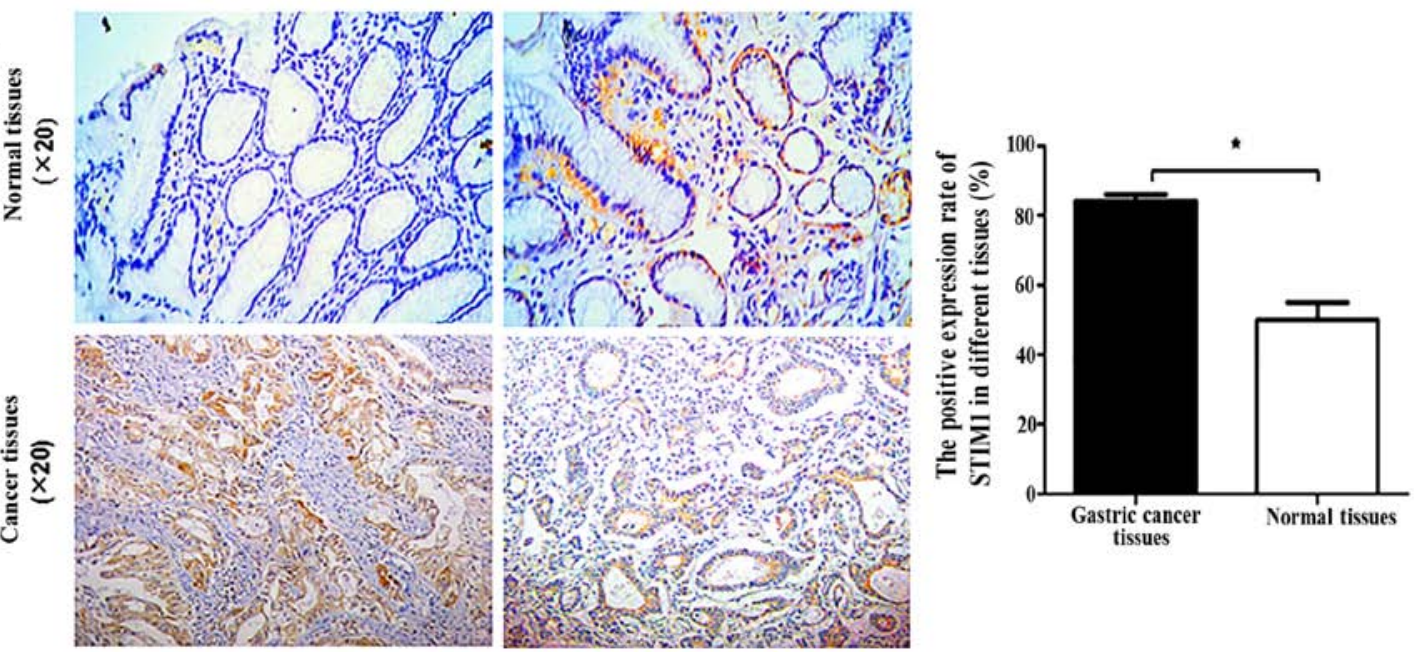

B Gastric cancer tissues
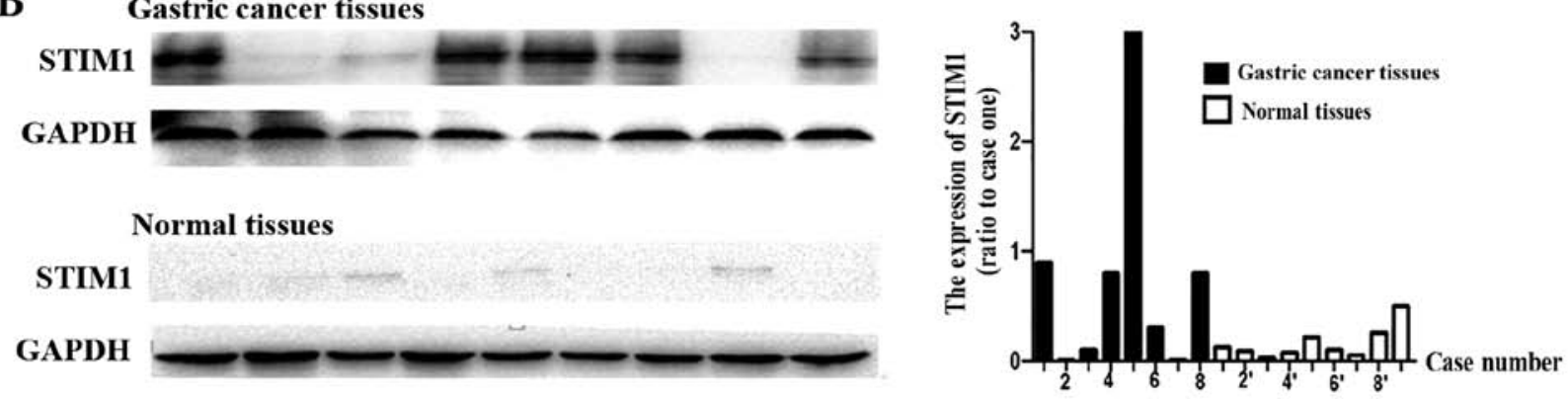

Figure 1. STIM1 expression in gastric cancer and normal gastric tissues. (A) Paraffin-embedded sections of gastric cancer and normal gastric tissues were collected. STIM1 expression was detected by immunohistochemistry using monoclonal mouse anti-STIM1 antibodies. ${ }^{*}$ P $<0.05$, vs. normal gastric tissues; Student's t-test. (B) Protein levels of STIM1 in fresh gastric cancer samples were analyzed by western blotting.

resuspended in $0.1 \% \mathrm{BSA}-1640$, and plated in the upper chambers in duplicate. The lower chamber was filled with RPMI-1640 medium containing 10\% FBS. After $24 \mathrm{~h}$, the non-migrating cells were removed from the upper membrane surface with a cotton swab. Transwell membranes were then fixed with $4 \%$ paraformaldehyde and stained with crystal violet. The number of cells that migrated through to the lower side of the membrane was counted under a light microscope with a 20 or $40 x$ objective. The invasion assay was performed using the ECM550 (Chemicon, Shanghai, USA) as an index of the invasive activity of tumor cells. FBS $(10 \%)$ was added to the lower chamber, as described for the cell migration experiments, and cell counting was performed as described for the cell migration experiments.

Adhesion assay. After transfection, cells $\left(2 \times 10^{5}\right.$ cells $\left./ \mathrm{ml}\right)$ were seeded in 96-well plates pre-incubated with Matrigel for $20 \mathrm{~min}$ at $37^{\circ} \mathrm{C}$. Unattached cells were removed by washing with PBS. Attached cells were fixed in $4 \%$ paraformaldehyde for $15 \mathrm{~min}$ and stained with $0.02 \%$ crystal violet solution for $15 \mathrm{~min}$. Randomly chosen fields were photographed under a light microscope with a 20 or $40 x$ objective.

Statistical analysis. All data are shown as the means \pm standard deviations (SDs). Statistical analyses were performed using the Statistical Package for Social Science program (SPSS for Windows, version 13.0; SPSS, Inc., Chicago, IL, USA) based on the results of three independent experiments. One-way analysis of variance (ANOVA) was used for comparing differences between groups. Differences with P-values of $<0.05$ were considered to be statistically significant.

\section{Results}

STIM1 is expressed at higher levels in gastric tumor tissues than that in normal gastric tissues. To clarify the clinical relevance of STIM1 expression in gastric cancer progression, 90 gastric tumor and 30 normal gastric tissues were analyzed by immunohistochemistry. Additionally, 8 fresh gastric tumor tissues and 9 fresh normal gastric tissues were analyzed by western blotting. STIM1 was expressed in both gastric cancer and normal gastric tissues (Fig. 1). The rate of STIM1 positivity was higher in the gastric cancer tissues than the rate in normal gastric tissues (Fig. 1A). Although STIM1 immunoreactivity was found in normal tissues, enhanced immunoreactivity was detected in the cancer tissues (Fig. 1B). These results confirmed that STIM1 was more frequently expressed in gastric cancer tissues than in normal tissues.

STIM1 and pathological factors. To analyze the relationship between STIM1 expression and clinical stages of gastric cancer, gastric cancer tissue samples were grouped according to patient age, gender, clinical and $\mathrm{T}$ stages, and differentiation (Table I). The rate of STIM1 positivity for gastric cancers 
A

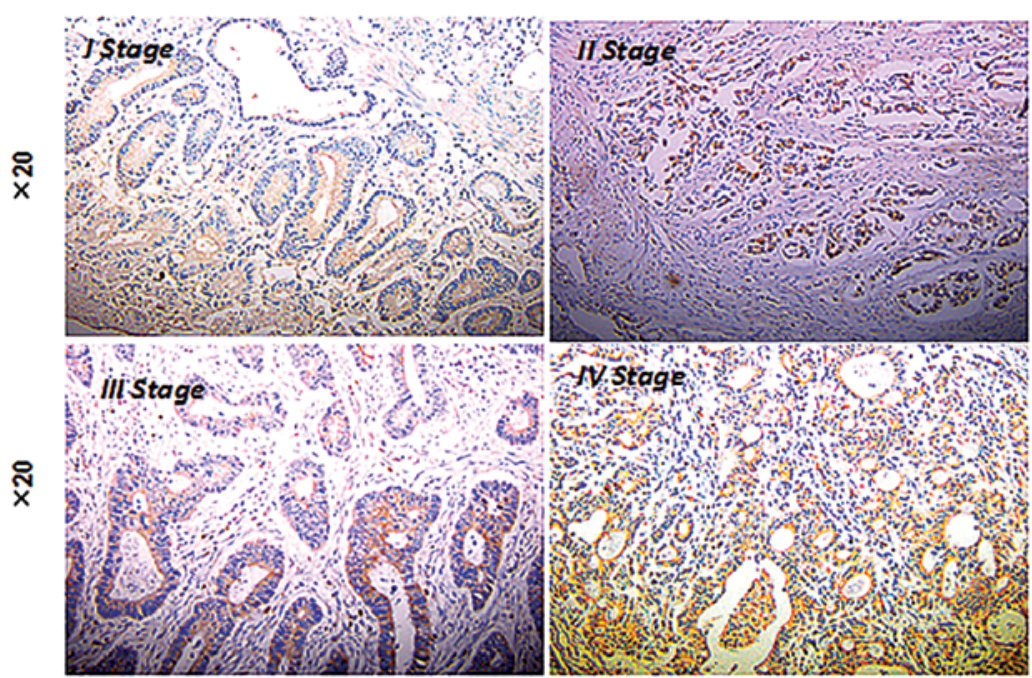

\section{C}

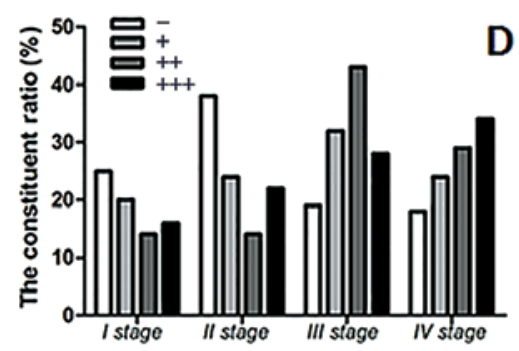

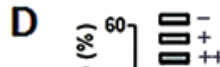

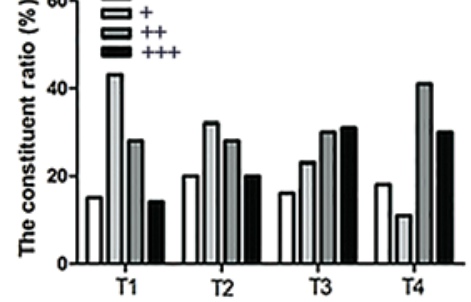

B

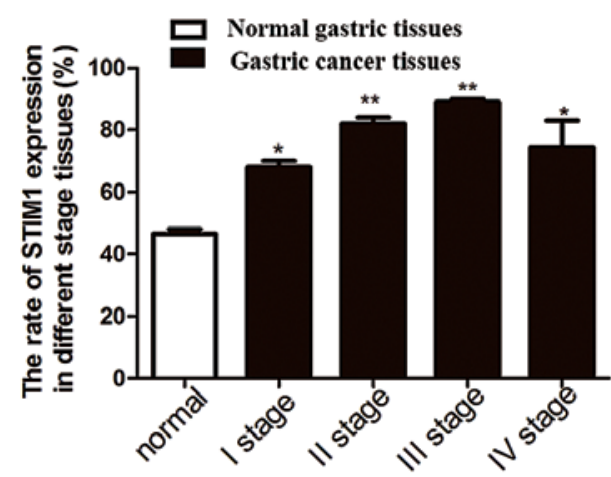

E

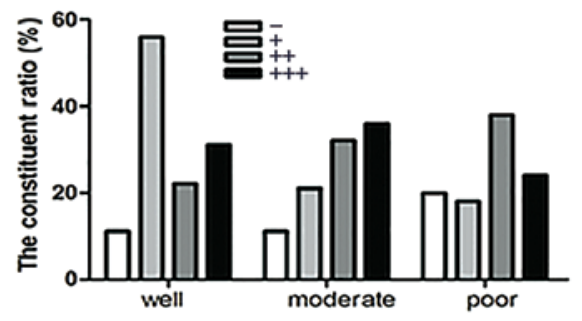

Figure 2. STIM1 and pathological factors. (A) The expression of STIM1 was detected by immunohistochemistry gastric cancer tissues of different clinical stages. Staining of four representative tissues is shown: I, II, III and IV stage. (B) Rates of STIM1 positivity in patients with different clinical stages of gastric cancer and non-cancerous tissues. ${ }^{*} \mathrm{P}<0.05,{ }^{* *} \mathrm{P}<0.01$ vs. normal gastric tissues; Student's t-test. The constituent ratio of STIM1 expression (negative, weak, moderate and strong) in four clinical stages (C), four T stages (D), and three differentiation levels (E) of gastric cancer.

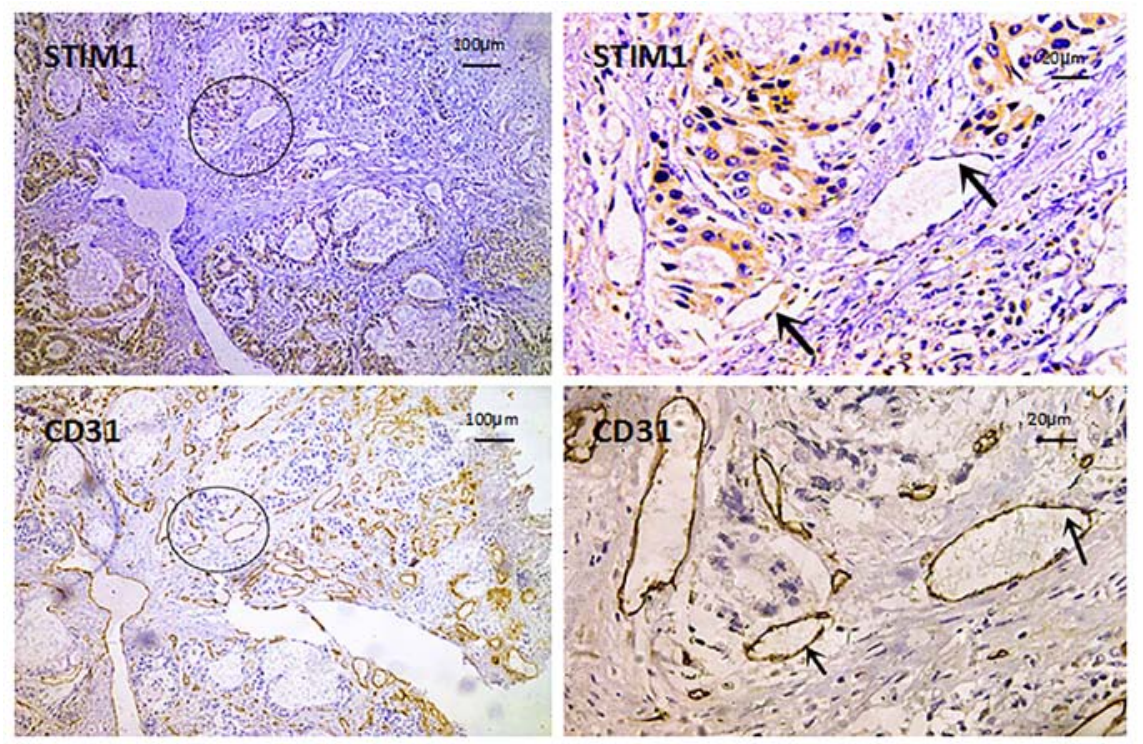

Figure 3. STIM1 expression in tumor endothelial cells in gastric tumor tissues. Tissues were double stained for CD31 as a marker of tumor vascular endothelial cells and STIM1 using immunohistochemistry. Cells that stained for both STIM1 and CD31 are indicated by black arrows.

of all stages was higher than the rate in normal gastric tissues (Fig. 2A and B). However, differential expression was not observed among the different clinical stages (Fig. 2B). STIM1 expression in patients with clinical stage I tended to be weaker than that in patients with clinical stages II, III or IV (Fig. 2C), suggesting that STIM1 expression may increase as the cancer becomes more advanced. Additionally, STIM1 expression was increased with increasing $\mathrm{T}$ stage (Fig. 2D). 
A

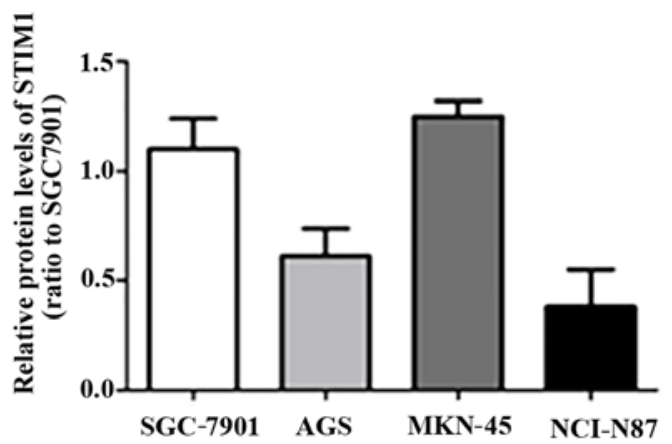

B
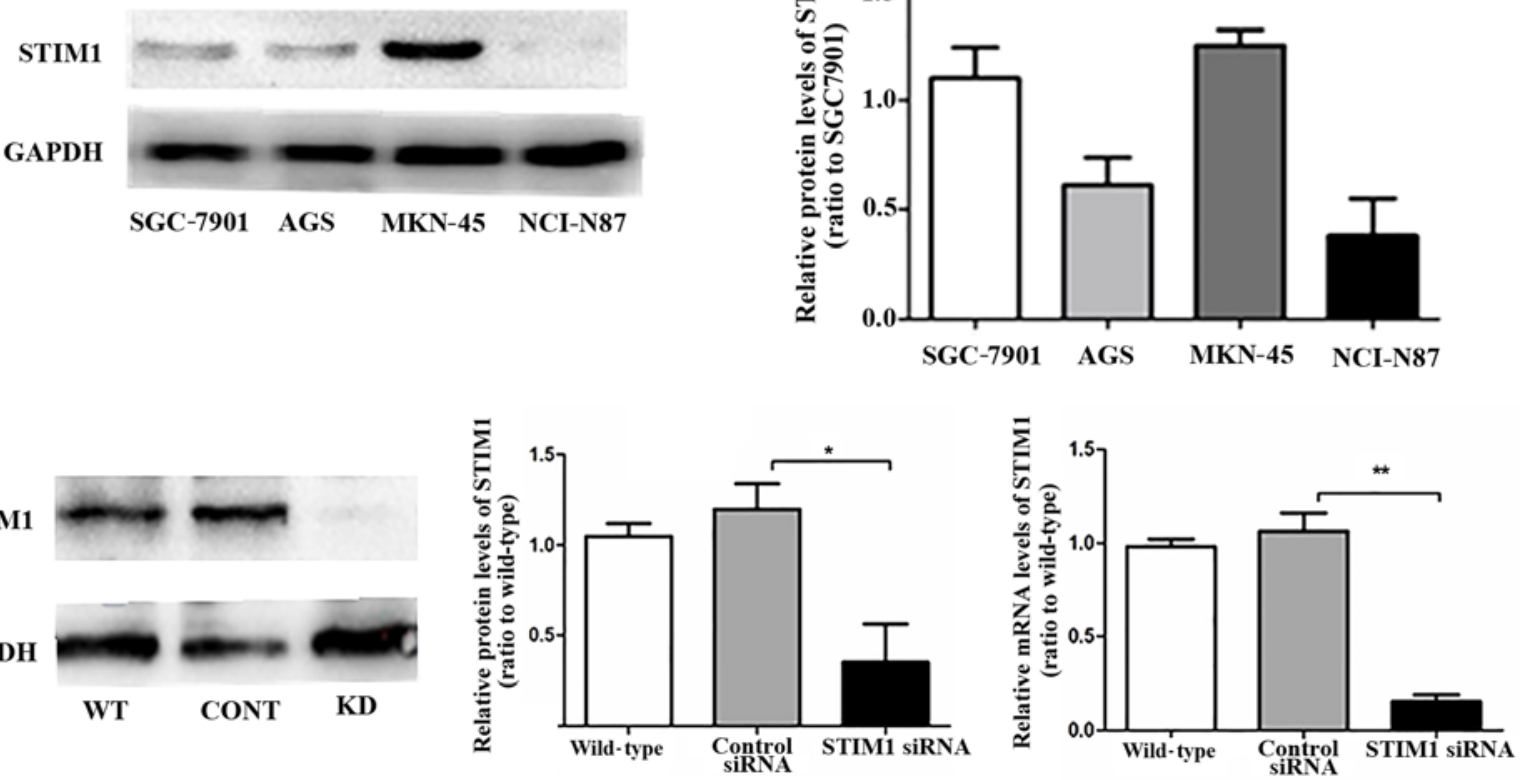

Figure 4. Expression of STIM1 in four gastric cancer cell lines. (A) Western blotting was used to detect STIM1 expression in four gastric cancer cell lines. (B) Efficiency of STIM1 knockdown by siRNA in gastric cancer cells. STIM1 mRNA and protein levels were measured by RT-PCR and western blotting, respectively, after transfection with siRNA. All values represent the means \pm SDs of three independent experiments. ${ }^{*} \mathrm{P}<0.05,{ }^{* *} \mathrm{P}<0.01$ vs. control siRNA; Student's t-test.

Next, we measured changes in STIM1 expression in tumor cells and normal cells with varying degrees of differentiation, as a measure of the degree of malignancy. STIM1 expression also increased as the degree of differentiation decreased (Fig. 2E). Therefore, taken together, these data suggest that STIM1 expression is associated with the degree of malignancy in gastric cancer.

We then analyzed the expression of CD31 as a molecular marker of endothelial cells using immunohistochemistry. We found that STIM1-positive cells colocalized with CD31-positive cells, suggesting that tumor vascular endothelial cells express STIM1 (Fig. 3).

STIM1 is expressed in four gastric cancer cell lines. We next analyzed the expression of STIM1 in MKN-45, SGC-7901, AGS and NCI-N87 cells. MKN-45 and SGC-7901 cells exhibited similar expression levels of STIM1, >2-fold higher than that in the NCI-N87 cells (Fig. 4A). Notably, both of these cell lines expressing high levels of STIM1 also showed high migration capacity. In preparation for our next experiments, we then examined the effects of an siRNA targeting STIM1 on STIM1 expression in MKN-45 cells. Western blotting and RT-PCR showed that siRNA targeting STIM1 effectively downregulated expression of STIM1 (Fig. 4B).

STIM1 does not affect proliferation or apoptosis in gastric cancer cells. As shown, using MTT assays, transfection with STIM1 siRNA did not affect the proliferation of the MKN-45 and SGC-7901 cells (Fig. 5B). Additionally, there were no statistically significant differences in the numbers of apoptotic cells after transfection with control siRNA or siRNA targeting STIM1, as measured using Annexin V-FITC/PI staining with flow cytometry (Fig. 5A).
Table I. Relationships between STIM1 staining intensity and pathological patient features.

\begin{tabular}{|c|c|c|c|c|c|}
\hline \multirow[b]{2}{*}{ Feature } & \multicolumn{4}{|c|}{ Immunoreactive score } & \multirow[b]{2}{*}{ Tota } \\
\hline & - & + & ++ & +++ & \\
\hline Age (years) & & & & & 78 \\
\hline$<40$ & 0 & 0 & 0 & 1 & 1 \\
\hline $40-55$ & 7 & 4 & 4 & 3 & 18 \\
\hline $56-65$ & 5 & 5 & 7 & 12 & 29 \\
\hline$>65$ & 1 & 7 & 15 & 7 & 30 \\
\hline Gender & & & & & 79 \\
\hline Male & 7 & 11 & 20 & 19 & 57 \\
\hline Female & 7 & 5 & 6 & 4 & 22 \\
\hline Clinical stage & & & & & 87 \\
\hline I & 4 & 6 & 3 & 3 & 16 \\
\hline II & 5 & 6 & 8 & 6 & 25 \\
\hline III & 4 & 4 & 12 & 8 & 28 \\
\hline IV & 3 & 4 & 5 & 6 & 18 \\
\hline T stage & & & & & 92 \\
\hline $\mathrm{T} 1$ & 1 & 3 & 2 & 1 & 7 \\
\hline $\mathrm{T} 2$ & 5 & 8 & 6 & 5 & 25 \\
\hline $\mathrm{T} 3$ & 7 & 10 & 13 & 13 & 43 \\
\hline $\mathrm{T} 4$ & 3 & 2 & 7 & 5 & 17 \\
\hline Differentiation & & & & & 62 \\
\hline Well & 1 & 5 & 2 & 1 & 9 \\
\hline Moderate & 2 & 4 & 6 & 7 & 19 \\
\hline Poor & 7 & 6 & 13 & 8 & 34 \\
\hline
\end{tabular}

STIM1, stromal interaction molecule 1. 
A

MKN-45
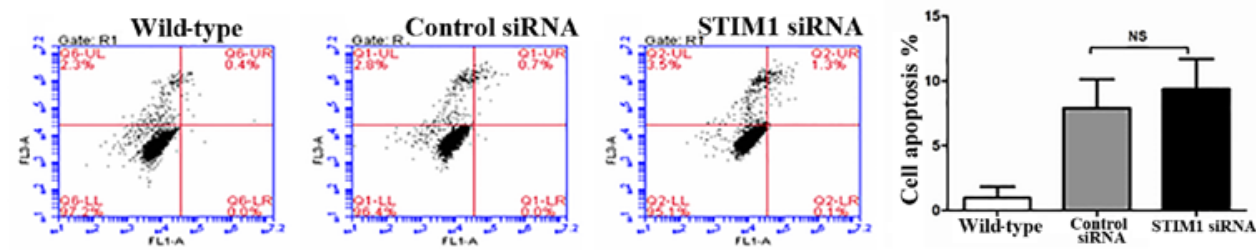

SGC-7901
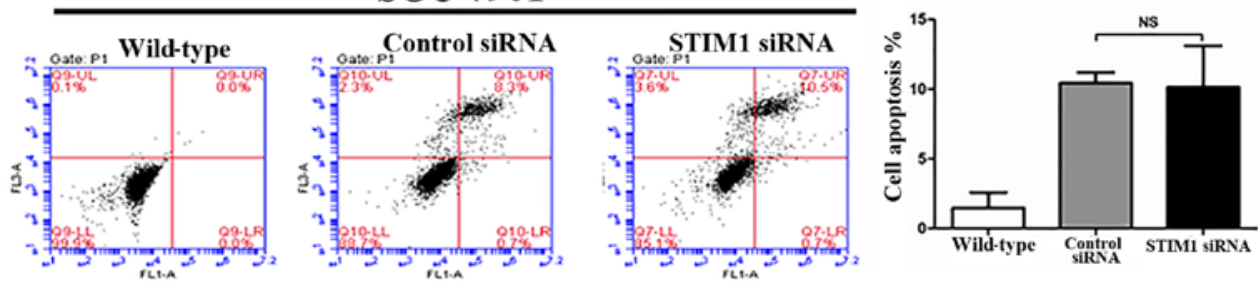

B
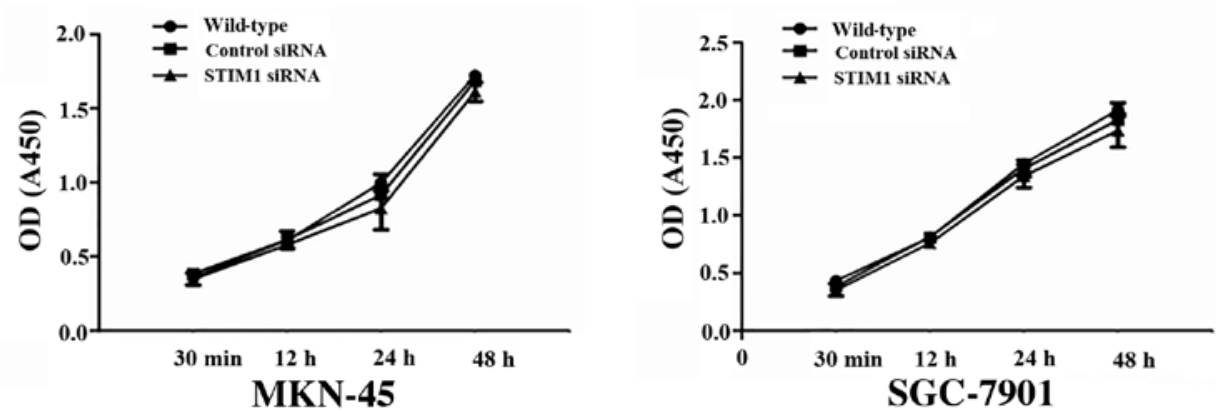

Figure 5. Effects of STIM1 knockdown on gastric cancer cell proliferation and apoptosis. (A) Cell apoptosis was measured by flow cytometry following Annexin V-FITC/PI staining. NS, P>0.05 vs. control siRNA in Student's t-test. (B) Gastric cancer cell proliferation was measured using MTT assays after transfection with STIM1 siRNA. All values represent the means \pm SD of three independent experiments.
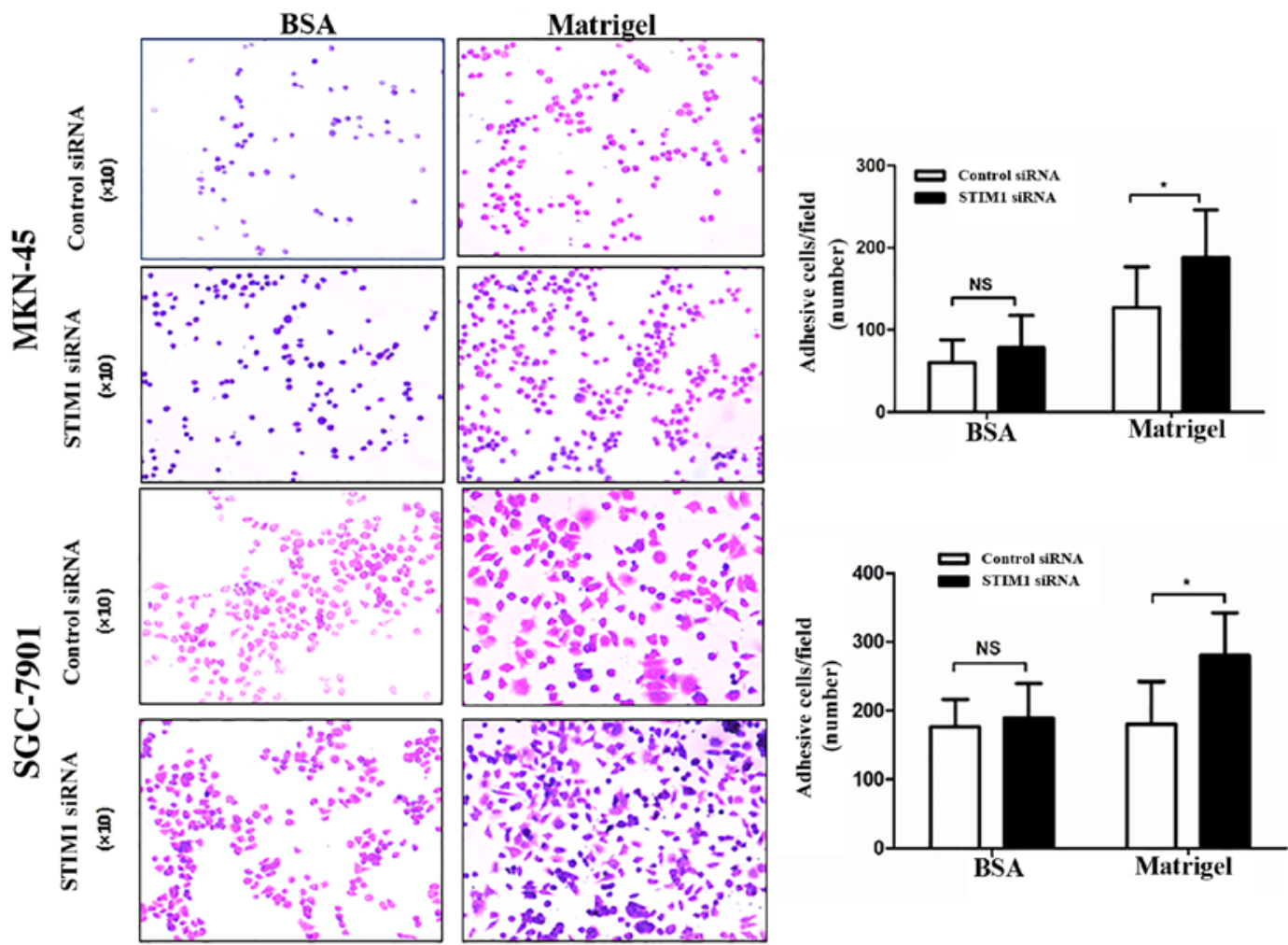

Figure 6. STIM1 knockdown affects cell adhesion. Cells $\left(2 \times 10^{5}\right.$ cells $\left./ \mathrm{ml}\right)$ were transfected and seeded in 96-well plates coated with Matrigel. Unattached cells were removed by washing with PBS, and plates were stained with $0.02 \%$ crystal violet solution for 15 min. Attached cells were counted under a light microscope with a 20 or $10 \mathrm{X}$ objective. All values represent the means \pm SD of three independent experiments. NS, P $>0.05$; ${ }^{*} \mathrm{P}<0.05$ vs. control siRNA in Student's t-test. 

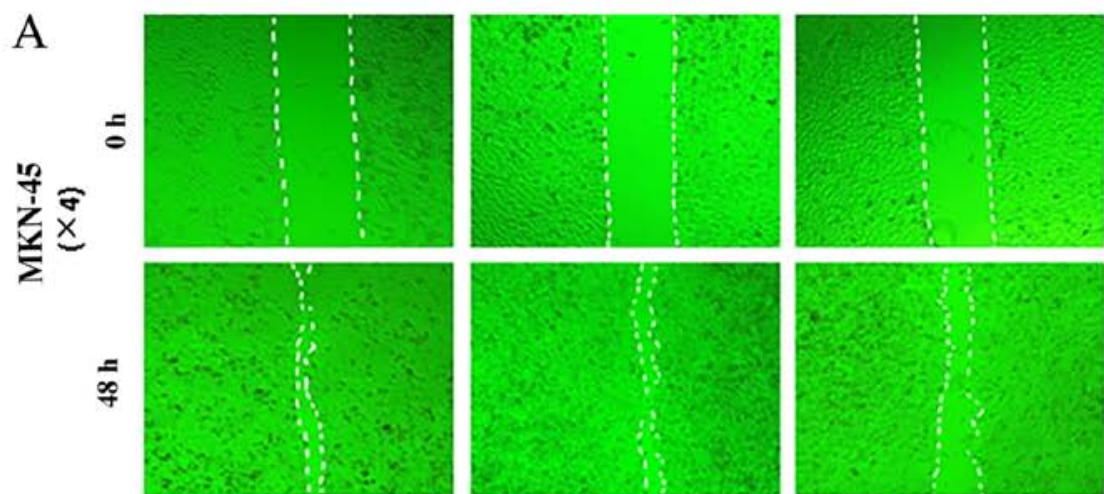

$\mathrm{C}$
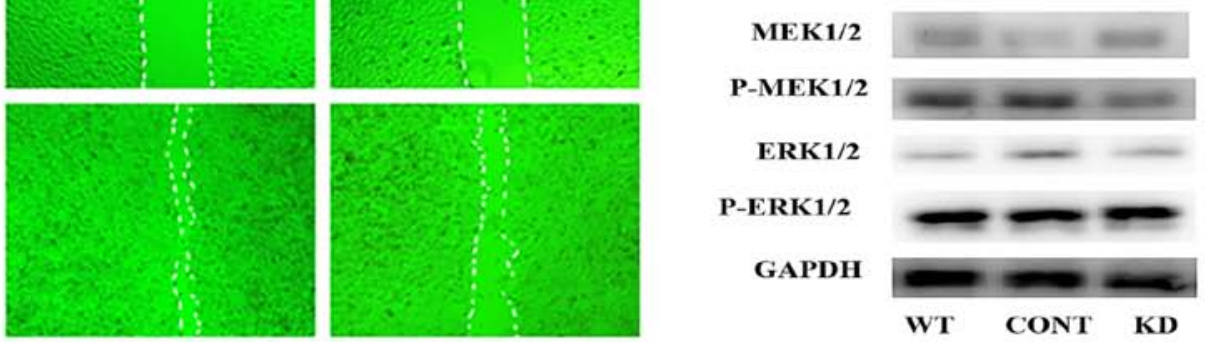

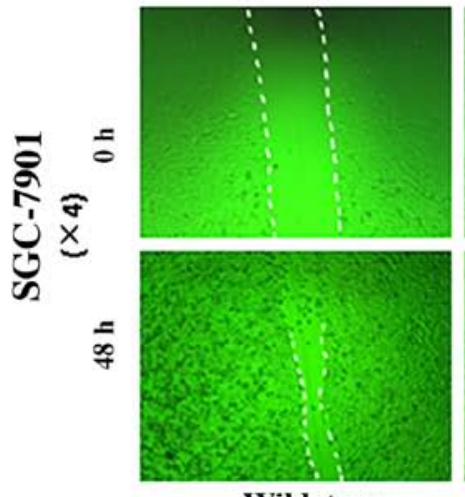

Wild-type

B
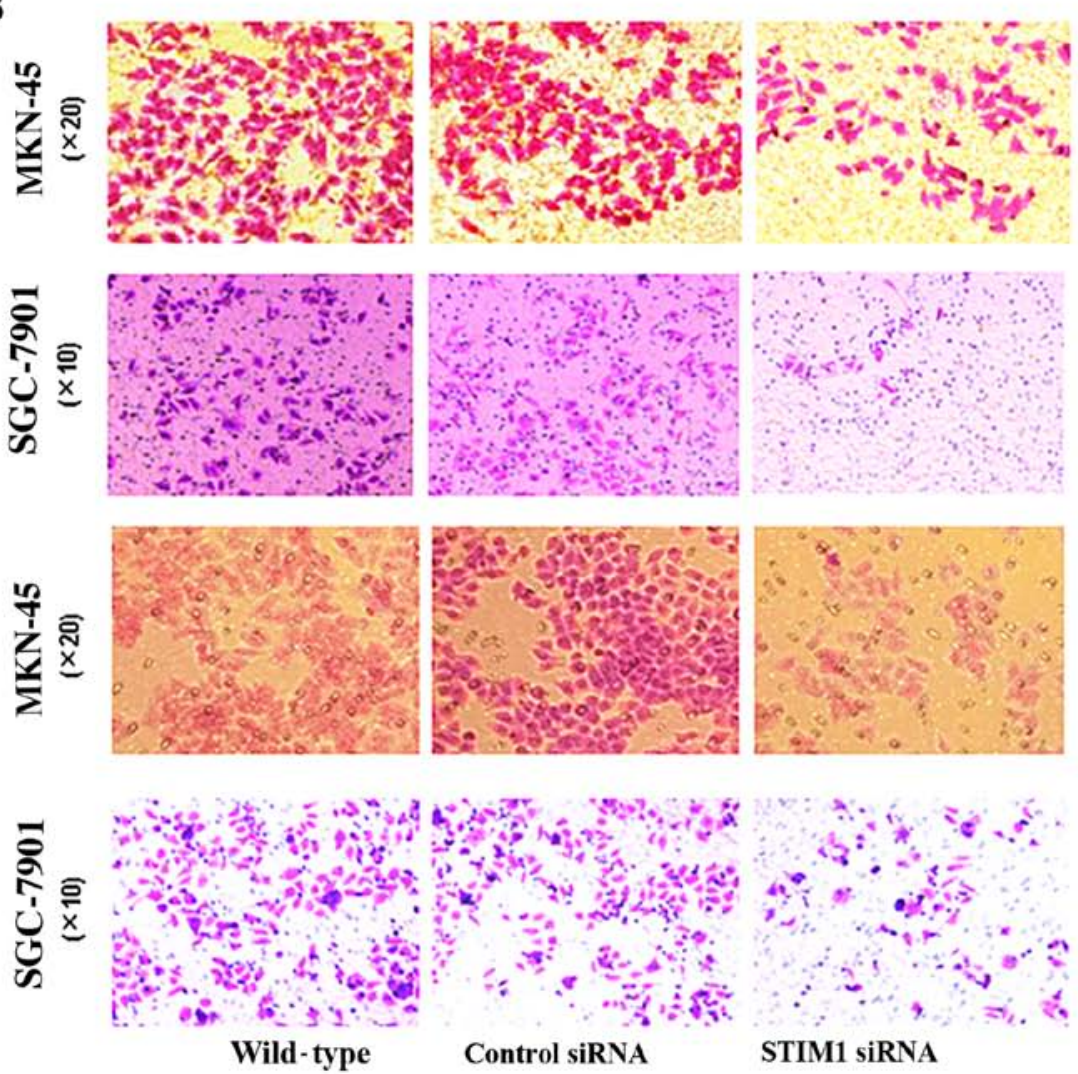
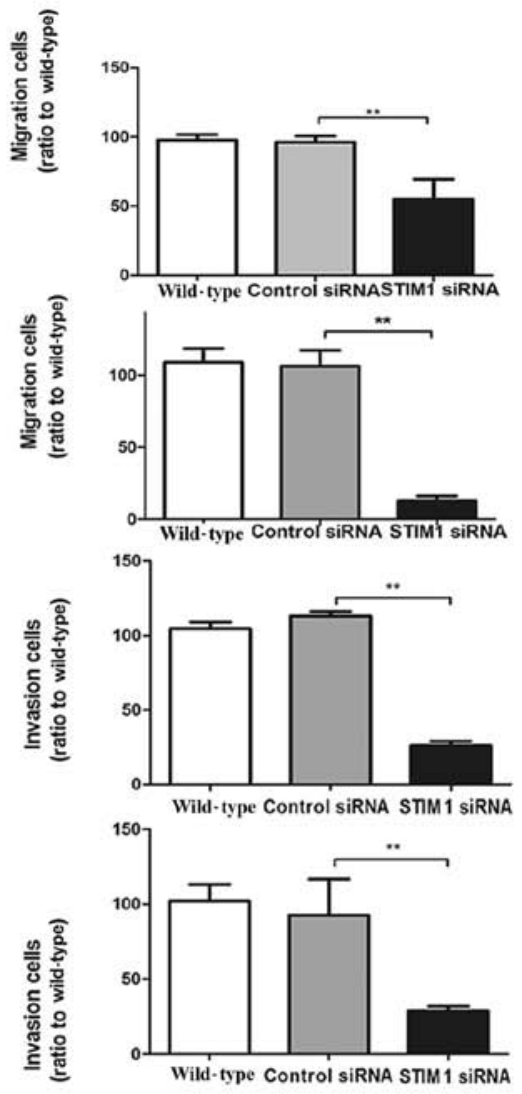

Figure 7. STIM1 knockdown reduces gastric cancer cell migration and invasion. (A) Wound healing assay. Cells were transfected with control or STIM1 siRNA and wound healing assays were performed. The width of the wound was measured after $48 \mathrm{~h}$. (B) Transwell migration and invasion assays were performed using MKN45 and SGC7901 cells following transfection with control or STIM1 siRNA. ${ }^{* *} \mathrm{P}<0.01$ vs. control siRNA in Student's t-test. (C) The involvement of STIM1 in the MEK/ERK signaling pathway was analyzed by western blotting. All values represent the means $\pm \mathrm{SD}$ of three independent experiments. NS, P $>0.05$.

STIM1-knockdown affects the migration and invasion of gastric cancer cells. We then examined the adhesion capacity of gastric cancer cells after transfection with STIM1 siRNA using Matrigel-coated plates. Matrigel simulates extracellular 
matrix (ECM) components and can be used to study adhesion and cell motility. In our assay, transfection with STIM1 siRNA enhanced the adhesion ability of the cells in the Matrigelcoated wells but did not affect adhesion in the wells coated with $0.1 \%$ bovine serum albumin (Fig. 6), indicating that knockdown of STIM1 promoted the ability of gastric cancer cells to detach from the ECM.

Next, we examined the effects of STIM1 knockdown on cell migration and invasion, two process that are critical for tumor metastasis. In the wound healing assay, the width of the wound in the cells transfected with STIM1 siRNA was greater than that in the control cells. As shown in Fig. 7B, knockdown of STIM1 by siRNA in the MKN-45 and SGC-7901 cells reduced cell migration through membranes compared with the migration assay of the control siRNA-transfected cells. Furthermore, invasion assays using Transwell chambers demonstrated that STIM1 knockdown also blocked cell invasion (Fig. 7A). Taken together, these results showed that STIM1 plays an important role in controlling tumor cell motility.

Finally, we further examined the mechanisms through which STIM1 mediated cell metastasis by evaluating changes in the phosphorylation of MEK/ERK proteins, which are involved in regulating cell division, differentiation, proliferation and motility. Western blotting showed that STIM1 knockdown did not alter the expression or phosphorylation of MEK or ERK (Fig. 7C). Therefore, STIM1 was involved in regulation of motility in gastric cancer cells through a method independent of the MEK/ERK pathway.

\section{Discussion}

In the present study, we examined the role of STIM1 in gastric cancer progression and metastasis. Our results showed that STIM1 was highly expressed in gastric cancer cell lines and tissues and that knockdown of STIM1 enhanced adhesion and reduced metastasis-related cellular functions, including migration adn invasion. Therefore, these data suggest that STIM1 may represent a novel target in the treatment of gastric cancer.

Tumor growth and metastasis are closely related to tumor angiogenesis. Several studies have shown that anti-angiogenic agents can inhibit tumor growth and distant metastasis. Moreover, STIM1 is expressed in human umbilical vein endothelial cells (HUVECs) and pulmonary artery endothelial cells (HPAECs), promoting endothelial cell proliferation and regulating the function of endothelial tissue, which is involved in angiogenesis $(18,19)$. Our results also found that STIM1 was expressed in tumor vascular endothelial cells. Therefore, the effects of STIM1 on the promotion of gastric cancer metastasis may be associated with the regulation of tumor angiogenesis.

Of the four gastric cancer cell lines examined in the present study, the highest level of STIM1 expression was observed in the MKN-45 cells, which were also the most highly metastatic cells. Consistent with the importance of STIM1 in cancer, STIM1 has been suggested to be a tumor suppressor, inducing cell death in rhabdomyosarcoma and rhabdoid tumor cell lines, which do not express endogenous STIM1 (20). In contrast, breast cancer cell lines express easily detectable levels of STIM1 mRNA, and no relationship between STIM1 expression and proliferation or apoptosis was observed in breast cancer cell lines. However, in other studies, STIM1 has been shown to mediate cell proliferation. Indeed, STIM1 knockdown has been shown to decrease $\mathrm{p} 21$ and $\mathrm{Cdc} 25 \mathrm{C}$ protein levels in cervical cancer, whereas several cell cycle regulators, including cyclin A and B1 and Cdk1, were not affected by STIM1 knockdown (16). Numerous studies have shown that STIM1 is involved in tumor cell apoptosis in response to anticancer drugs $(16,21)$. However, our results did not support a direct role for STIM1 in gastric cancer cell apoptosis, and knockdown of STIM1 did not affect the proliferation of the MKN-45 or SGC-7901 cells. Therefore, the role of STIM1 in cancer cell proliferation may vary according to cell type.

In the present study, we also examined the effects of STIM1 on cancer cell migration and invasion, which are related to the metastatic potential of cancer cells and can be used to distinguish between benign and malignant tumors. In previous studies, Yang et al (13) demonstrated that silencing of STIM1 or Orail inhibited the migration and metastasis of breast cancer cells by suppressing focal adhesion turnover. Moreover, reduction of STIM1 by RNA interference or application of a pharmacological inhibitor of SOCE promoted intercellular adhesion and weakened tumor cell invasion and migration in hepatic carcinoma (15). In the present study, we found that knockdown of STIM1 inhibited gastric cancer cell migration and invasion and promoted adhesion. These effects may be mediated through extracellular matrix (ECM) components. Cell migration is an integrated process requiring both tumor cells and the surrounding ECM, and we hypothesized that STIM1 may promote gastric cancer metastasis through interaction with integrins as part of the ECM. Alternatively, metastasis is a complex, highly regulated process that also involves various intracellular signaling pathways, including the MEK/ERK pathway, which regulates cell division, differentiation, proliferation and motility (22-25). For example, Ono et al (26) and Neuzillet et al (27) reported that silencing of PTK6 reduces ERK1/2 activation, whereas PTK6 overexpression increased ERK1/2 activation, thereby promoting cellular migration and invasion in pancreatic cancer. Additionally, Nagini (28) found that vascular endothelial growth factor (VEGF) expression was increased when the ERK signaling pathway was inhibited. In the present study, we showed that knockdown of STIM1 did not alter the expression or activation of MEK or ERK, implying that STIM1 affected gastric cancer cell migration through a pathway independent of the MEK/ ERK pathway.

In conclusion, our results showed that STIM1 was expressed both in gastric cancer and normal gastric tissues, with more frequent expression in cancerous tissues than in normal tissues. STIM1 did not affect proliferation or apoptosis in gastric cancer cells. However, knockdown of STIM1 blocked cell migration and invasion and promoted cell adhesion. Further studies are required to determine the mechanisms involved. We hypothesized that STIM1, which promotes the proliferation of endothelial cells, may affect cancer metastasis by regulating angiogenesis. Whether there is a different level of STIM1 in tumor vascular endothelial and normal vascular endothelial cells warrants further investigation. This hypothesis will need to be tested in further in-depth studies. In any case, our results provide a basis for the further analysis of STIM1 as a specific molecular target in cancer therapy. 


\section{Acknowledgements}

The present study was supported by the National Clinical Medicine Research Foundation of China (grant no. L2012055), and the National Natural Science Foundation of China (grant no. 81302170$)$.

\section{References}

1. Ferlay J, Shin HR, Bray F, Forman D, Mathers C and Parkin DM: Estimates of worldwide burden of cancer in 2008: GLOBOCAN 2008. Int J Cancer 127: 2893-2917, 2010.

2. Berridge MJ, Bootman MD and Roderick HL: Calcium signalling: Dynamics, homeostasis and remodelling. Nat Rev Mol Cell Biol 4: 517-529, 2003.

3. Zheng L, Stathopulos PB, Li GY and Ikura M: Biophysical characterization of the EF-hand and SAM domain containing $\mathrm{Ca}^{2+}$ sensory region of STIM1 and STIM2. Biochem Biophys Res Commun 369: 240-246, 2008.

4. Oh-Hora M, Yamashita M, Hogan PG, Sharma S, Lamperti E, Chung W, Prakriya M, Feske S and Rao A: Dual functions for the endoplasmic reticulum calcium sensors STIM1 and STIM2 in T cell activation and tolerance. Nat Immunol 9: 432-443, 2008

5. Hewavitharana T, Deng X, Soboloff J and Gill DL: Role of STIM and Orai proteins in the store-operated calcium signaling pathway. Cell Calcium 42: 173-182, 2007.

6. Muik M, Schindl R, Fahrner M and Romanin C: $\mathrm{Ca}^{2+}$ releaseactivated $\mathrm{Ca}^{2+}$ (CRAC) current, structure, and function. Cell Mol Life Sci 69: 4163-4176, 2012

7. Stathopulos PB, Zheng L, Li GY, Plevin MJ and Ikura M: Structural and mechanistic insights into STIM1-mediated initiation of store-operated calcium entry. Cell 135: 110-122, 2008.

8. Omilusik KD, Nohara LL, Stanwood S and Jefferies WA: Weft, warp, and weave: The intricate tapestry of calcium channels regulating T lymphocyte function. Front Immunol 4: 164, 2013.

9. Matsumoto $\mathrm{M}$ and Baba Y: Role of STIM-dependent $\mathrm{Ca}^{2+}$ influx in regulatory B cells. Yakugaku Zasshi 133: 419-425, 2013 (In Japanese).

10. Félix R, Crottès D, Delalande A, Fauconnier J, Lebranchu Y, Le Guennec JY and Velge-Roussel F: The Orai-1 and STIM-1 complex controls human dendritic cell maturation. PLoS One 8 e61595, 2013.

11. Lee KJ, Woo JS, Hwang JH, Hyun C, Cho CH, Kim do H and Lee EH: STIM1 negatively regulates $\mathrm{Ca}^{2+}$ release from the sarcoplasmic reticulum in skeletal myotubes. Biochem J 453: 187-200, 2013.

12. Fodor J, Matta C, Oláh T, Juhász T, Takács R, Tóth A, Dienes B, Csernoch L and Zákány R: Store-operated calcium entry and calcium influx via voltage-operated calcium channels regulate intracellular calcium oscillations in chondrogenic cells. Cell Calcium 54: 1-16, 2013.

13. Yang S, Zhang JJ and Huang XY: Orai1 and STIM1 are critical for breast tumor cell migration and metastasis. Cancer Cell 15: 124-134, 2009.
14. Chen YF, Chiu WT, Chen YT, Lin PY, Huang HJ, Chou CY, Chang HC, Tang MJ and Shen MR: Calcium store sensor stromal-interaction molecule 1-dependent signaling plays an important role in cervical cancer growth, migration, and angiogenesis. Proc Natl Acad Sci USA 108: 15225-15230, 2011.

15. Yang N, Tang Y, Wang F, Zhang H, Xu D, Shen Y, Sun S and Yang G: Blockade of store-operated $\mathrm{Ca}^{2+}$ entry inhibits hepatocarcinoma cell migration and invasion by regulating focal adhesion turnover. Cancer Lett 330: 163-169, 2013.

16. Sun S, Li W, Zhang H, Zha L, Xue Y, Wu X and Zou F. Requirement for store-operated calcium entry in sodium butyrate-induced apoptosis in human colon cancer cells. Biosci Rep 32: 83-90, 2012

17. Suyama E, Wadhwa R, Kaur K, Miyagishi M, Kaul SC, Kawasaki $\mathrm{H}$ and Taira K: Identification of metastasis-related genes in a mouse model using a library of randomized ribozymes. J Biol Chem 279: 38083-38086, 2004.

18. Abdullaev IF, Bisaillon JM, Potier M, Gonzalez JC, Motiani RK and Trebak M: Stim1 and Orai1 mediate CRAC currents and store-operated calcium entry important for endothelial cell proliferation. Circ Res 103: 1289-1299, 2008.

19. Lodola F, Laforenza U, Bonetti E, Lim D, Dragoni S, Bottino C, Ong HL, Guerra G, Ganini C, Massa M, et al: Store-operated $\mathrm{Ca}^{2+}$ entry is remodelled and controls in vitro angiogenesis in endothelial progenitor cells isolated from tumoral patients. PLoS One 7: e42541, 2012.

20. Sabbioni S, Barbanti-Brodano G, Croce CM and Negrini M: GOK: A gene at $11 \mathrm{p} 15$ involved in rhabdomyosarcoma and rhabdoid tumor development. Cancer Res 57: 4493-4497, 1997.

21. Li W, Zhang M, Xu L, Lin D, Cai S and Zou F: The apoptosis of non-small cell lung cancer induced by cisplatin through modulation of STIM1. Exp Toxicol Pathol 65: 1073-1081, 2013.

22. Cerne JZ, Stegel V, Gersak K and Novakovic S; Jasmina-Ziva: $K R A S$ rs61764370 is associated with HER2-overexpressed and poorly-differentiated breast cancer in hormone replacement therapy users: A case control study. BMC Cancer 12: 105, 2012.

23. Sui JQ, Xie KP, Zou W and Xie MJ: Emodin inhibits breast cancer cell proliferation through the ER $\alpha$-MAPK/Akt-cyclin D1/Bcl-2 signaling pathway. Asian Pac J Cancer Prev 15: 6247-6251, 2014.

24. Zhong LR, Chen X and Wei KM: Radix tetrastigma hemsleyani flavone induces apoptosis in human lung carcinoma a549 cells by modulating the MAPK pathway. Asian Pac J Cancer Prev 14: 5983-5987, 2013.

25. de Mello RA, Marques DS, Medeiros R and Araújo AM: Epidermal growth factor receptor and K-Ras in non-small cell lung cancer-molecular pathways involved and targeted therapies. World J Clin Oncol 2: 367-376, 2011.

26. Ono H, Basson MD and Ito H: PTK6 promotes cancer migration and invasion in pancreatic cancer cells dependent on ERK signaling. PLoS One 9: e96060, 2014.

27. Neuzillet C, Hammel P, Tijeras-Raballand A, Couvelard A and Raymond E: Targeting the Ras-ERK pathway in pancreatic adenocarcinoma. Cancer Metastasis Rev 32: 147-162, 2013.

28. Nagini S: Carcinoma of the stomach: A review of epidemiology, pathogenesis, molecular genetics and chemoprevention. World $\mathrm{J}$ Gastrointest Oncol 4: 156-169, 2012. 